\title{
Développement territorial intégré et leadership locaux
}

\author{
René Lachapelle, professionnel de recherche et Denis Bourque, titulaire \\ Chaire de recherche du Canada en organisation communautaire \\ Université du Québec en Outaouais
}

\section{INTRODUCTION}

Cet article s'appuie sur une démarche en cours de recherche inductive ${ }^{1}$ sur le développement territorial intégré sur la base de monographies d'expériences locales et régionales de concertation pour le développement ${ }^{2}$. Quatre de ces monographies d'expériences de concertation, utilisant la recherche documentaire et des entrevues avec des acteurs engagés, ont été publiées par la Chaire de recherche du Canada en organisation communautaire. Elles présentent les démarches de concertation de la MRC Les Moulins, de COSMOSS dans le Bas-SaintLaurent, du développement local au Sénégal et du développement social sur la Côte-Nord. Une cinquième monographie est en rédaction sur la politique de développement social de la MRC de Vaudreuil-Soulanges. L'état actuel des travaux permet d'en dégager, d'une part, un constat commun quant à l'importance de la dimension territoriale dans les démarches d'action concertée pour le développement; d'autre part, l'identification de profils de leadership assumés par les divers acteurs du développement dont celui des professionnels qui agissent en soutien à ces démarches quel que soit leur titre d'emploi.

L'article s'attache donc à présenter dans un premier temps la notion de développement territorial intégré (DTI) telle qu'elle se dégage de la littérature récente sur le territoire et l'action collective laquelle connaît une multiplication de ses formes depuis quelques années nécessitant d'identifier des caractéristiques et des indicateurs permettant de mieux reconnaître ce qui relève du DTI. Sur cette base, la seconde partie de l'article présente le contexte de nouvelle action publique dans lequel s'inscrivent les initiatives de DTI et les divers leaderships assumés par les acteurs qui y participent. L'article se conclut sur le leadership de processus que l'on désigne aussi comme une fonction de soutien assumée par les divers agents de développement dans les démarches de DTI.

\section{LE DÉVELOPPEMENT TERRITORIAL INTÉGRÉ (DTI)}

\section{Le territoire représente à la fois un cadre d'action réunissant les gens concernés par une situation problématique et le résultat de cette action. Cette perspective est caractéristique de l'organisation communautaire quelle que soit l'approche dans laquelle elle s'inscrit.}

Le territoire comme construit à partir d'«un concours d'acteurs, dans un contexte spatial déterminé qui vise à faire émerger, puis à tenter de résoudre, sous contrainte environnementale, un problème sociétal ou productif partagé. » Considéré comme un système d'acteurs situés dans l'espace et le temps, le territoire représente à la fois un cadre d'action réunissant les gens concernés par une situation problématique et le résultat de cette action. Cette perspective est caractéristique de l'organisation communautaire quelle que soit l'approche dans laquelle elle s'inscrit'.

Au cours des trois dernières décennies, on a assisté à l'émergence de démarches concertées sur des bases sectorielles (développement économique local, enjeux sociaux particuliers des jeunes, des familles, des aînés, etc.). Au cours de la dernière décennie, les programmes d'action publique et les fondations philanthropiques ont repris ce modèle et suscité nombre de concertations, dites intersec- 
torielles, autour de leurs propres objectifs. Cette multiplication de démarches concertées a entrainé l'hyperconcertation et, de ce fait, soulevé l'enjeu de l'intégration des démarches de développement sur une base territoriale: est-ce que le territoire d'appartenance des acteurs partenaires d'un projet concerté ne pourrait pas devenir la clé d'une intégration des actions autour de la réponse à la problématique d'un milieu donné?

\section{Le développement territorial intégré (DTI) repose sur la mobilisation des forces sociales d'un milieu et des ressources auxquelles il a accès, dans un cadre qui fait tomber les cloisonnements sectoriels et qui ouvre les programmes aux besoins locaux.}

Les auteurs considèrent que le développement ne saurait permettre une véritable avancée collective s'il n'est pas à la fois économique et social et s'il n'arrive pas à produire de nouvelles réponses aux besoins collectifs qui demeurent insatisfaits lorsque l'on s'en tient à la traditionnelle croissance du PIB. Une telle intégration de l'économique et du social à laquelle on pourrait ajouter la prise en compte des enjeux environnementaux et de la culture de chaque collectivité dans une perspective de développement durable, exige des acteurs qu'ils inscrivent leur action propre dans un projet collectif d'amélioration du bien vivre ${ }^{4}$. Ils doivent donc d'abord partager un diagnostic commun de la situation territoriale en termes de problèmes à régler, mais aussi de leviers locaux qu'ils sont en mesure de mettre à contribution; il leur faut ensuite renouveler les rapports entre les acteurs afin de les ouvrir à l'innovation en associant les populations exclues aux démarches de développement. Le développement territorial intégré (DTI) repose sur la mobilisation des forces sociales d'un milieu et des ressources auxquelles il a accès, dans un cadre qui fait tomber les cloisonnements sectoriels et qui ouvre les programmes aux besoins locaux.

À quel moment un système local d'action atteint-il un stade de DTI? Quelles sont les composantes essentielles d'un tel système pour qu'un milieu s'engage dans l'innovation sociale? En s'appuyant sur 86 recherches portant sur des expériences partenariales réalisées dans une dizaine d'États membres de l'Union européenne, une étude propose quatre caractéristiques clés fournissant «une définition fonctionnelle spécifique du partenariat local $»$ :

- une structure formelle pour l'élaboration des décisions et leur mise en œuvre;

- la mobilisation d'une coalition d'intérêts et l'engagement de différents partenaires;

- un ordre du jour et un programme d'action multidimensionnel communs;

- un but ou une finalité qui est de lutter contre le chômage, la pauvreté et l'exclusion sociale ainsi que de promouvoir la cohésion sociale et l'intégration.

Cette structure organisationnelle qui constitue le moyen de mise en œuvre du DTI, demeure en elle-même de l'ordre de la planification stratégique appliquée à un territoire. Pour identifier son apport concret au «chassé-croisé d'acteurs locaux » lui permettant de devenir une démarche innovante de DTI, trois niveaux de résultats sont proposés par Geddes :

- l'instauration d' " un esprit de collaboration » entre les acteurs locaux;

- l'association de l'ensemble des acteurs locaux à la démarche, y compris les « groupes exclus »;

- l'adaptation des programmes «aux capacités et aux besoins locaux » et la défense des intérêts locaux.

Il identifie aussi deux limites importantes: «Plus les problèmes d'une région sont d'ordre structurel $[\ldots]$ moins le partenariat local en soi ne sera en mesure d'apporter des solutions » et le fait «que les partenariats sont très difficiles à instituer dans un contexte local où il n'existe aucun environnement sociopolitique solidaire $»^{5}$.

Ces conclusions correspondent à celles de l'équipe québécoise de recherche sur 10 initiatives locales de lutte à la pauvreté, qui identifie «trois éléments cruciaux » en termes de modes de fonctionnement pour réaliser un système local de DTI: «1) l'insertion des leaders dans de multiples réseaux de nature territoriale et sectorielle; 2) la stabilité du leadership afin de développer une connaissance des réseaux et des 
ressources existantes; 3) l'ouverture et la capacité de partager le savoir-faire acquis ». On parle $\mathrm{d}$ '« une construction sociale où dispositions personnelles, formations professionnelles et expériences de terrain se combinent en un ensemble de capacités permettant de surmonter des tensions, des problèmes, des crises et des situations de concurrence entre organisations afin de générer des compromis, des consensus et des alliances ». Les acteurs qui interagissent dans l'espace local, contribuent à construire un leadership collectif ancré dans la territorialité et en même temps générateur d'un renouvellement de cette appartenance territoriale. Ainsi, en organisant l'action sur une base territoriale, on incite la population de ce territoire à s'identifier comme acteur et on construit une appartenance, un "nous" autour de cette action".

\section{Les acteurs qui interagissent dans l'espace local, contribuent à construire un leadership collectif ancré dans la territorialité et en même temps générateur d'un renouvellement de cette appartenance territoriale.}

Nous retenons quatre indicateurs permettant de reconnaître une démarche comme étant de type DTI :

- la territorialisation de l'activité économique et sociocommunautaire et la prise en compte de la dimension environnementale du développement;

- la structuration d'un système local d'action concertée, c'est-à-dire un mécanisme reposant sur la concertation comme processus collectif de coordination basé sur une mise en relation structurée et durable entre des acteurs sociaux autonomes qui acceptent de partager de l'information, de discuter de problèmes ou d'enjeux spécifiques à un territoire afin de convenir d'objectifs et d'action en commun;

- une démonstration de résultats locaux non seulement en termes de partage d'information, mais aussi d'actions manifestant l'accroissement de la capacité d'agir locale;
- une participation citoyenne incluant les populations exclues.

Ces indicateurs ne se manifestent pas partout de la même façon ni avec la même intensité, mais on devrait pouvoir les retrouver partout où il est question de DTI.

La territorialisation est un indicateur de processus. Elle devrait se traduire par l'établissement sur la base d'un territoire de liens entre les acteurs économiques des secteurs privé, public et d'économie sociale et solidaire, mais aussi par des liaisons entre l'activité économique, l'action sociocommunautaire et l'action environnementale. En ce sens, la territorialisation dépasse l'intersectorialité entre acteurs institutionnels et communautaires. Des initiatives locales dont le niveau d'intégration est variable peuvent être considérées comme territoriales dans la mesure où elles entraînent un dépassement des confinements sectoriels qui caractérisent le modèle gestionnaire libéral.

Le second indicateur, plus immédiatement vérifiable, renvoie à une structure dont les activités manifestent le caractère fonctionnel. Les expériences locales et régionales adoptent une diversité de modèles, plus ou moins inclusifs, de concertation axée sur le développement d'un territoire. Pour qu'un système local d'action concertée ait un caractère intégrateur, il doit au minimum permettre la présence simultanée de représentants d'institutions, d'élus et d'initiatives de la société civile (organismes communautaires, mouvements sociaux, etc.) et aussi favoriser l'articulation des liens avec les acteurs, les programmes et les instances externes au territoire, mais qui le déterminent dans son développement.

\section{La territorialisation est un indicateur de} processus. Elle devrait se traduire par l'établissement sur la base d'un territoire de liens entre les acteurs économiques des secteurs privé, public et d'économie sociale et solidaire, mais aussi par des liaisons entre l'activité économique, l'action sociocommunautaire et l'action environnementale. 
Les résultats sont certainement l'indicateur le plus difficile à utiliser, car les démarches de développement opèrent sur un horizon de moyen et surtout de long terme, la durée étant un facteur déterminant ${ }^{6}$. En même temps, elles doivent éviter un reproche fréquent à l'effet que la concertation s'en tient à des activités d'échange d'information sans vraiment générer d'actions concrètes. D'autres chercheurs ont montré que plusieurs initiatives de concertation n'arrivent pas à dépasser ce niveau du partage d'information. Ces démarches de concertation ne sont pas moins utiles puisqu'elles permettent des liaisons entre intervenants, mais une concertation doit afficher une action concrète, sans quoi elle peut difficilement prétendre être agent de développement ${ }^{8}$.

\section{LES PRATIQUES LOCALES D'ACTION CONCERTÉE}

\section{Les systèmes locaux d'action concertée sont efficaces lorsqu'ils permettent d'harmoniser l'exercice de leaderships diversifiés par des acteurs dont les intérêts ne sont pas nécessairement convergents. À travers cette coopération conflictuelle se développe une capacité d'agir et émerge une appartenance commune à un territoire comme espace d'intégration des logiques ascendante et descendante.}

Au Québec, les pratiques de DTI se déploient dans le cadre de systèmes locaux d'action concertée (SLAC) dans lesquels sont à l'œuvre des logiques paradoxales ${ }^{3}$. Une logique descendante, d'une part, portée par l'État et de grands bailleurs de fonds, partagée entre le respect de la participation démocratique des communautés et une stratégie de gestion technocratique centrée sur les objectifs et les règles de programmes; une logique ascendante, d'autre part, issue de la capacité d'initiative des acteurs locaux, principalement communautaires, partagée entre la demande de respect de l'autonomie et de l'identité, et la demande de reconnaissance et d'intégration aux politiques et programmes publics. Ces SLAC sont des espaces de négociation entre des programmes favorisant à divers degrés leur appropriation par les milieux et des initiatives communautaires plus ou moins disposées à la complémentarité.
La pierre d'achoppement de toutes les démarches concertées est de ne réunir que des intervenantes et intervenants sans inclure directement des citoyennes et citoyens concernés par le développement recherché. Le DTI doit faire une différence pour les personnes, les groupes et les communautés appauvries ou exclues en termes de participation non seulement aux bénéfices du développement recherché, mais aussi à sa définition. Les organismes d'action communautaire autonome sont des lieux d'inclusion sociale qui jouent à cet égard un rôle déterminant dans les concertations locales. Leur contribution au caractère intégré du développement territorial est éminente.
Certains programmes utilisent les communautés pour atteindre leurs fins et certains organismes sont disposés à un rôle complémentaire pour obtenir des ressources alors que d'autres acteurs communautaires tiennent à leur autonomie et veulent soumettre les programmes à leurs propres objectifs.

Les systèmes locaux d'action concertée sont efficaces lorsqu'ils permettent d'harmoniser l'exercice de leaderships diversifiés par des acteurs dont les intérêts ne sont pas nécessairement convergents. À travers cette coopération conflictuelle se développe une capacité d'agir et émerge une appartenance commune à un territoire comme espace d'intégration des logiques ascendante et descendante. Cette « construction des liens entre les citoyens, entre les organisations, entre les institutions pour libérer les potentiels d'action d'une communauté territoriale constitue en fait un défi organisationnel important $»^{9}$.

\subsection{Nouvelle action publique et intégration des programmes}

La conjoncture actuelle est marquée par la création en 2003 des conférences régionales des élus (CRÉ) (Lois de 2003, chapitre 29, art. 9799). Cette décision marque un virage centralisateur dans le champ du développement territorial. Relevant du ministère des Affaires 
municipales, des Régions et de l'Occupation du territoire (MAMROT), les CRÉ sont « l'interlocuteur privilégié du gouvernement en matière de développement régional » (LRQ, M-22.1, art. 21.6). Leur mandat principal est «d'évaluer les organismes de planification et de développement au palier local et régional, dont le financement provient en tout ou en partie du gouvernement, de favoriser la concertation des partenaires dans la région et de donner, le cas échéant, des avis au ministre sur le développement de la région» (LRQ, M-22.1, art.21.7). Remplaçant des conseils régionaux de développement, les CRÉ donnent aux élus locaux une place prépondérante et marquent une réduction du rôle de la société civile dans la coordination régionale des initiatives de développement. C'est par les CRÉ que passent maintenant la plupart des ententes par lesquelles le gouvernement confie aux élus locaux la mise en œuvre de programmes, dont le Plan d'action gouvernemental pour la solidarité et l'inclusion sociale ${ }^{10}$.

Désigné par le sigle PAGSIS, ce second plan d'action gouvernemental en application de la Loi visant à lutter contre la pauvreté et l'exclusion sociale (LRQ, L-7) vise à «briser les "silos" », « assouplir les normes », « donner aux acteurs régionaux et locaux les moyens de mieux réaliser leur mission » et "assurer la continuité des démarches ». Pour ce faire, Québec « entend signer des Alliances pour la solidarité avec chacune des régions », leur confiant «la responsabilité de réunir les partenaires [...] afin d'établir un mécanisme de concertation à large spectre ». Les actions attendues visent à «valoriser et favoriser l'autonomie des personnes », «soutenir le revenu des personnes défavorisées » et enfin « améliorer les conditions de vie des personnes et des familles à faible revenu ». Les déterminants sociaux identifiés sont le logement, la sécurité alimentaire, les services de garde, l'offre de transport et « une meilleure compréhension des réalités propres aux autochtones du Québec ${ }^{10}$.

Le programme est soutenu par le Fonds québécois d'initiatives sociales doté de «115 millions pour les cinq années d'application du Plan» afin de soutenir à tous les paliers la «mobilisation et la concertation des acteurs », 1 '« approfondissement des connaissances » et le « soutien à des initiatives » (Idem : 20). Mais le contrôle de toute la démarche demeure entre les mains de l'administration puisque «le gouvernement confie aux conférences administratives régionales [...] la responsabilité d'assurer la cohérence de l'action gouvernementale en région» et «d'établir les liens entre les organismes gouvernementaux et les partenaires réunis par les Conférences régionales des élus » ${ }^{10}$

La dimension territoriale présente au niveau de la gouverne du programme repose sur une prépondérance de la logique descendante et limite la participation communautaire. Malgré la volonté affirmée d'«assurer la continuité des démarches» et de «partir des structures de concertation et de coordination déjà en place », certaines régions ont créé de nouvelles instances. Quant aux enjeux de développement territorial (logement, sécurité alimentaire, services de garde et transport), ils peuvent aisément être abordés de façon sectorielle plutôt qu'intégrée.

Les programmes publics auxquels on pourrait ajouter ceux qui proviennent des sociétés de gestion créées en partenariat avec la Fondation Lucie-et-André-Chagnon, illustrent bien la nouvelle action publique. L'État québécois modifie son rapport aux territoires et aux organismes de la société civile en installant une hiérarchie administrative, depuis les ministères jusqu'aux municipalités régionales de comté et aux grandes agglomérations, au moyen d'une instance régionale confiée à des élus municipaux. Les représentants des organisations de la société civile qui avaient initié les conseils régionaux de développement et la plupart des instances de concertation locale créées avant 1996, n'y participent que sur invitation et à la discrétion des élus.

Les collectivités locales voient arriver les ressources dont elles ont besoin, dans le cadre de programmes assortis de leur propre mode d'emploi. On crée de nouvelles structures qui, dans certains cas, font disparaître des concertations qui avaient fait leurs preuves, et, dans 
d'autres cas, s'ajoutent aux initiatives en place, contribuant à l'hyperconcertation ${ }^{3}$. Malgré la volonté affichée de sortir des logiques sectorielles qui bloquent un DTI, la nouvelle action publique en néglige une composante essentielle. En laissant moins de place aux organismes de la société civile, elle affaiblit la capacité d'innovation des agents de changement que sont les mouvements sociaux (Moulaert et Nussbaumer, 2008). L'innovation sociale passe en effet par la capacité des acteurs locaux à infléchir l'action en fonction de leurs propres objectifs. Pour y arriver ils doivent bâtir leur cohésion et faire preuve de leadership collectif, une tâche nettement plus complexe dans le cadre de la nouvelle action publique.

\subsection{Les systèmes locaux d'action concertée}

\section{Les systèmes locaux d'action concertée mettent à contribution divers types d'acteurs collectifs. D'une part, les institutions représentées par les directions locales; les élus qui assument le pouvoir politique local ; et les experts qui rendent accessibles les savoirs.}

Les systèmes locaux d'action concertée mettent à contribution divers types d'acteurs collectifs. D'une part, les institutions représentées par les directions locales; les élus qui assument le pouvoir politique local; et les experts qui rendent accessibles les savoirs. D'autre part, les citoyennes et citoyens assurent l'enracinement collectif des décisions concertées, et les organismes communautaires porteurs d'initiatives locales. S'ajoute, au niveau du soutien aux SLAC, le leadership de processus des intervenantes et intervenants dont nous traiterons en conclusion.

Le leadership institutionnel appartient aux directions des institutions et organismes assumant à l'échelle locale les missions sectorielles de 1'État: dans le secteur économique, les centres locaux de développement (CLD), les corporations de développement économique communautaire (CDEC) et les sociétés d'aide au développement des collectivités (SADC); dans le secteur de l'éducation et de la formation de la main-d'œuvre, les commissions scolaires et les cégeps, les centres locaux d'emploi (CLE) et les carrefours jeunesse emploi (CJE); dans le secteur social, les centres de santé et de services sociaux (CSSS). Ces institutions disposent de ressources publiques à l'échelon local et ont accès aux ressources régionales et nationales qu'elles peuvent mettre au service des besoins locaux. Leur défi c'est que les programmes appuient l'action locale plutôt que d'utiliser des énergies locales pour atteindre des fins administrativement déterminées. Cela ne va pas de soi pour des directions fortement incitées à demeurer dans la zone de confort des impératifs de gestion et des enjeux sectoriels comme on peut le constater dans plusieurs CSSS $^{3}$.

Le leadership expert est un volet des ressources institutionnelles : savoirs systématisés dans les banques de données; programmes de santé publique; «meilleures pratiques » appuyées sur des «données probantes »; modèles développés par des instituts ou des firmes; résultats de recherches académiques; etc. Les collectivités locales québécoises disposent actuellement de portraits de leur territoire aux plans économique et démographique (Statistique Canada et l'Institut de la statistique du Québec), de la main-d'œuvre (Emploi-Québec), des inégalités sociales et des problématiques communautaires (Santé et Services sociaux, MAMROT). Ces connaissances, même physiquement accessibles, peuvent facilement, dans leur forme savante, demeurer inutilisables par les communautés. Les professionnels doivent en faire la «traduction » pour servir les intérêts locaux.

Le leadership politique revient aux élus du fait de la légitimité que leur confère le statut de représentants de la population. Il leur revient de décider en dernière instance des choix collectifs. $\mathrm{Ce}$ pouvoir s'exerce aujourd'hui dans des domaines plus larges et plus exigeants que par le passé. Leur mandat ne se limite plus à l'entretien de la municipalité, mais s'étend à l'occupation et au développement du territoire. Leur légitimité démocratique ne leur confère pas sur ce plan la capacité d'en maîtriser les tenants et aboutissants. Ils ont intérêt à faire une place à la 
démocratie délibérative et à la participation citoyenne. Ils doivent donc apprendre à négocier avec les leaders communautaires et institutionnels, à reconnaître les expertises et à donner davantage de place aux citoyennes et citoyens. L'appareil administratif dont ils ont la responsabilité dispose de ressources déterminantes pour la mise en œuvre de projets locaux.

Le leadership communautaire est le fait d'organismes créés localement, en contact direct avec les citoyennes et citoyens. La politique de reconnaissance et de soutien à l'action communautaire (PRSAC) les présente comme des acteurs essentiels à l'exercice de la citoyenneté et au développement social du Québec.

Le leadership communautaire est le fait d'organismes créés localement, en contact direct avec les citoyennes et citoyens. La politique de reconnaissance et de soutien à l'action communautaire (PRSAC) les présente comme des acteurs essentiels à l'exercice de la citoyenneté et au développement social du Québec. Ils rassemblent les membres actifs de la communauté, dont plusieurs en situation de marginalité et de vulnérabilité ou désireux de faire valoir des droits sociaux. Ils contribuent à l'équité sociale, que ce soit par la revendication (action sociale), les services aux personnes (action sociocommunautaire), les initiatives de solidarité (éducation populaire, économie sociale, etc.) ou les alternatives communautaires (développement local). Dans les années 1980 et 1990, ils ont été les initiateurs des tables de concertation sectorielles et de fédérations multisectorielles que sont les corporations de développement communautaires (CDC). Les programmes qui misent sur l'action concertée, qu'ils proviennent d'institutions publiques ou de fondations privées, en font des partenaires souvent sur-sollicités, au risque de diluer leur capacité d'innovation. Le déplacement des ressources du soutien à la mission globale qui caractérise la PRSAC, vers des financements par projets affecte leur pérennité et leur marge d'autonomie. Le rattachement à un ministère a ramené une logique sectorielle qui ouvre la porte à ce déplacement malgré le maintien du financement à la mission globale sauf au ministère de 1'Emploi et de la Solidarité sociale (MESS) où dominent les ententes de services. L'affirmation du leadership communautaire face aux programmes ne va pas de soi en contexte de rareté des ressources.

Le leadership citoyen constitue le test de la participation. Les citoyennes, les citoyens sont des électeurs, des payeurs de taxes, des utilisateurs de services, des personnes qui occupent et construisent les espaces de proximité (voisinage, quartier, village, etc.) dans lesquels se fait le développement des territoires. Ce sont des acteurs essentiels à l'intégration territoriale. Collectivement, ils sont capables de contrebalancer la sensibilité à court terme des élus trop souvent alignés sur les échéances électorales, et les points de vue sectoriels des institutions et des organismes communautaires fortement conditionnés par les programmes assurant leur financement. Leur leadership repose sur l'information : plus elle est transparente, accessible et variée, plus elle alimente l'espace public et permet aux citoyennes et citoyens de former une opinion éclairée. Mais il passe aussi par la création d'espaces de prise de parole: assemblées publiques, rencontres de consultation, etc. Il n'y a pas de développement durable sans que la population n'endosse les choix collectifs.

\section{Le leadership citoyen constitue le test de la participation. Les citoyennes, les citoyens sont des électeurs, des payeurs de taxes, des utilisateurs de services, des personnes qui occupent et construisent les espaces de proximité (voisinage, quartier, village, etc.) dans lesquels se fait le développement des territoires.}

L'ensemble de ces leaderships est essentiel à la démocratie délibérative locale requise par le DTI. Pour qu'ils s'exercent, il faut un leadership rassembleur qui peut venir de n'importe quel groupe d'acteurs. Mais il faut aussi que la gouverne de la démarche reçoive le soutien convenable. C'est la responsabilité du leadership de processus. 


\section{LE LEADERSHIP DE PROCESSUS ET LE DOUBLE DÉFI D'INTÉGRER LES POPULATIONS EXCLUES ET DE FAIRE PASSER L'ACTION DES PLANS À LA RÉALISATION}

Le leadership de processus est le fait des intervenantes et intervenants responsables de soutenir les démarches d'un SLAC. Ces passeurs assurent la circulation de l'information et la liaison sur plusieurs registres entre les acteurs en présence: rendre accessibles les contenus experts; aider à comprendre le point de vue des autres acteurs; assurer le respect mutuel des cultures en présence; rendre effective la participation de tous quel que soit leur niveau de savoir et de pouvoir; structurer les rapports entre les logiques descendante et ascendante. Actuellement, au Québec, plusieurs intervenantes et intervenants professionnels assument ces fonctions au palier local: organisatrices et organisateurs communautaires des CSSS $( \pm 450)$, agents de développement rural des MRC ou des CLD $( \pm 180)$, auxquels s'ajoute un nombre non déterminé d'agents de liaison et d'agents de développement embauchés dans les organismes de développement territorial ou les structures issues des fondations. Ces métiers du développement local ${ }^{11}$ requièrent des compétences qui ne sont pas seulement académiques même si la formation initiale leur fournit des outils essentiels ${ }^{12}$. Leur leadership repose sur les compétences acquises dans l'action et sur une stabilité de présence dans les communautés.

La recherche dans la MRC Les Moulins a mis en évidence le manque de résultats générés par certaines concertations du fait que les «lieux de concertation soient trop axés sur l'échange et qu'ils ne transforment pas les discussions en actions $»^{8}$. Une démarche de DTI devient initiative de développement dans la mesure où elle réussit à générer des projets qui font reculer l'exclusion. Les inégalités sociales et l'affaiblissement du lien social entraînent la baisse de la participation citoyenne ${ }^{14}$. Instaurer des espaces de participation ne suffit pas pour restaurer la capacité de participer de personnes en situation de marginalité et d'exclusion. La politique québécoise de soutien à l'action communautaire reconnaît aux organismes communautaires une fonction de développement social, entre autres parce qu'ils sont capables de soutenir l'inclusion sociale. En permettant aux personnes appauvries de s'inscrire dans des relations égalitaires, ils contribuent à restaurer le tissu social. Cela peut se réaliser dans la revendication au nom des droits sociaux, économiques et environnementaux, mais aussi dans les approches d'organisation communautaire qui intègrent des processus de capacitation ou de conscientisation ${ }^{13}$. La participation des «premiers concernés » aux instances de concertation en DTI passe généralement par les organismes communautaires où se réalise l'empowerment permettant une participation aux instances de concertation. Avec les mouvements sociaux (syndical, féministe, écologiste, etc.), les groupes communautaires et les organismes d'économie sociale et solidaire jouent un rôle majeur de changement social en associant au DTI les personnes et les groupes sociaux dont les intérêts sont mal servis par le modèle dominant. Leur présence est essentielle pour obliger les systèmes locaux d'action concertée à prendre en compte la pauvreté et l'exclusion.

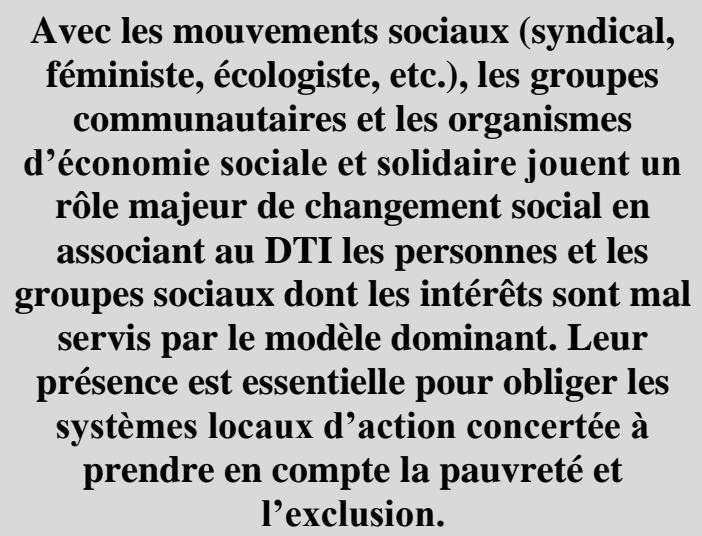

Plusieurs expériences affichent des résultats concrets correspondant aux indicateurs retenus. On peut y reconnaître une concrétisation de la «capacité de transformer des problèmes en projet collectif $»^{15}$. Les démarches d'action concertée donnent leur mesure lorsqu'elles réussissent à s'inscrire dans la durée. Il faut une certaine pérennité pour que les acteurs apprennent à dépasser les approches sectorielles et que les collectivités mobilisent leurs ressources locales et aillent chercher celles qui leur font défaut pour un développement intégré de leur territoire. 


\section{BIBLIOGRAPHIE}

${ }^{1}$ Albarello, Luc (2011), Choisir l'étude de cas comme méthode de recherche, Bruxelles : De Boeck, 144p.

Luckerhoff, Jason \& François Guillemette (sous la direction de) (2012), Méthodologie de la théorisation enracinée, Fondements, procédures et usages, Québec : Presses de l'Université du Québec, 282p.

${ }^{2}$ Pecqueur, Bernard \& Xabier Itçaina (2012), «Économie sociale et solidaire et territoire : un couple allant de soi? », Revue internationale de l'économie sociale Recma, no.325, juillet, p.48-64. Yin Robert K. (1994), Case Study Research: Design and Methods, $2 d$ ed., Thousand Oaks, CA: Sage Publications, 240p.

${ }^{3}$ Bourque, Denis (2008), Concertation et partenariat. Entre levier et piège du développement des communautés, Québec: Presses de l'Université du Québec, 142p. Bourque, Denis, Lachapelle, René, Savard, Sébastien, Tremblay, Marielle, \& Danielle Maltais (2010). Les effets de la création des CSSS sur les pratiques partenariales, psychosociales et communautaires, Rapport de recherche, Co-publication CRCOC ARUC-ISDC - GRIR - LAREPPS, décembre, 176p.

${ }^{4}$ Moulaert, Frank \& Jacques Nussbaumer (2008), La logique sociale du développement territorial, Québec: Presses de l'Université du Québec, 153p. Ndiaye, Sambou (2012), Le développement économique local au Sénégal: un état des lieux, Cahiers de la CRCOC, no. 1201, Université du Québec en Outaouais, 38p. Vaillancourt, Yves (2010), «Le projet de société alternatif en Équateur : Socialisme ou Social-démocratie $\mathrm{du} \mathrm{XXI}^{\mathrm{e}}$ siècle?', Vie économique, vol.2 no. $2 ., 13$ p.

${ }^{5}$ Geddes, Michael (1998), Partenariat local: une stratégie réussie pour la cohésion sociale? Dublin, Fondation européenne pour l'amélioration des conditions de vie et de travail - Luxembourg, Office des publications officielles des communautés européennes, 193p.

${ }^{6}$ Klein, Juan-Luis (2011), «Économie sociale et territoire en contexte de mondialisation : le développement par l'initiative locale» in Bellemare, Guy \& Klein, Juan-Luis (sous la direction de) (2011). Innovation sociale et territoire, Québec: Presses de l'Université du Québec, pp.175-194. Klein, Juan-Luis \& Christine Champagne (sous la direction de) (2011), Initiatives locales et lutte contre la pauvreté et l'exclusion, Québec : Presses de l'Université du Québec, 325p.

${ }^{7}$ RQIIAC (2010), Pratiques d'organisation communautaire en CSSS. Cadre de référence du RQIIAC, Québec, Presses de l'Université du Québec, 158p.

${ }^{8}$ Côté, Nathalie, St-Louis, Marie-Pierre \& Denis Bourque (2010). Pratiques de concertation sur le territoire de la MRC des Moulins : rapport de la démarche de recherche participative, Chaire de recherche du Canada en organisation communautaire, Cahier no. 1003, mai, 78p. ${ }^{9}$ Québec (2010), Le Québec mobilisé contre la pauvreté. Plan d'action gouvernemental pour la solidarité et l'inclusion sociale, Ministère de l'Emploi et de la Solidarité sociale, 54p.

${ }^{10}$ Caillouette Jacques, Boyer Ginette, Dallaire Nicole, Garon Suzanne \& Alex Ellyson (2007), « Le développement des communautés locales comme enjeu du renouvellement de l'intervention publique et communautaire », Intervention, no. 126, juin, pp.31-41.

${ }^{11}$ Robitaille, Martin (2007), Les métiers du développement local et régional au Québec: l'émergence de nouvelles compétences, Université du Québec en Outaouais - Co-publication de la Chaire de recherche en développement des collectivités et du Centre de recherche sur le développement territorial, Série: Recherches, no.9, février, 31p.

${ }^{12}$ Lachapelle, René \& Denis Bourque (2011), COSMOSS - Communauté ouverte et solidaire pour un monde outillé, scolarisé et en santé, Une démarche de concertation régionale en développement social, Cahiers de la CRCOC no. 1101, 55p.

${ }^{13}$ Freire, Paolo (1974), Pédagogie des opprimés suivi de Conscientisation et Révolution, Paris, François Maspero, 205p. Plamondon, Annie (2006), La lutte du Collectif pour une loi sur l'élimination de la pauvreté, Montréal, Cahiers du CRISES no. MS0704, 49p. Comeau, Yvan (2012), « Les stratégies d'intervention sociopolitique favorables à l'action collective ", Reflets : revue d'intervention sociale et communautaire, vol. 18, no. 1 (à paraître).

${ }^{14}$ Paugam, Serge (2008), Le lien social, Paris, Presses universitaires de France, 127p.

${ }^{15}$ Favreau, Louis \& Lucie Fréchette (2002), Organisation communautaire et intervention de quartier en Outaouais : l'expérience du quartier Jean Dallaire de Hull, CÉRIS-UQO, Cahier série Recherche no.22, 46p. 


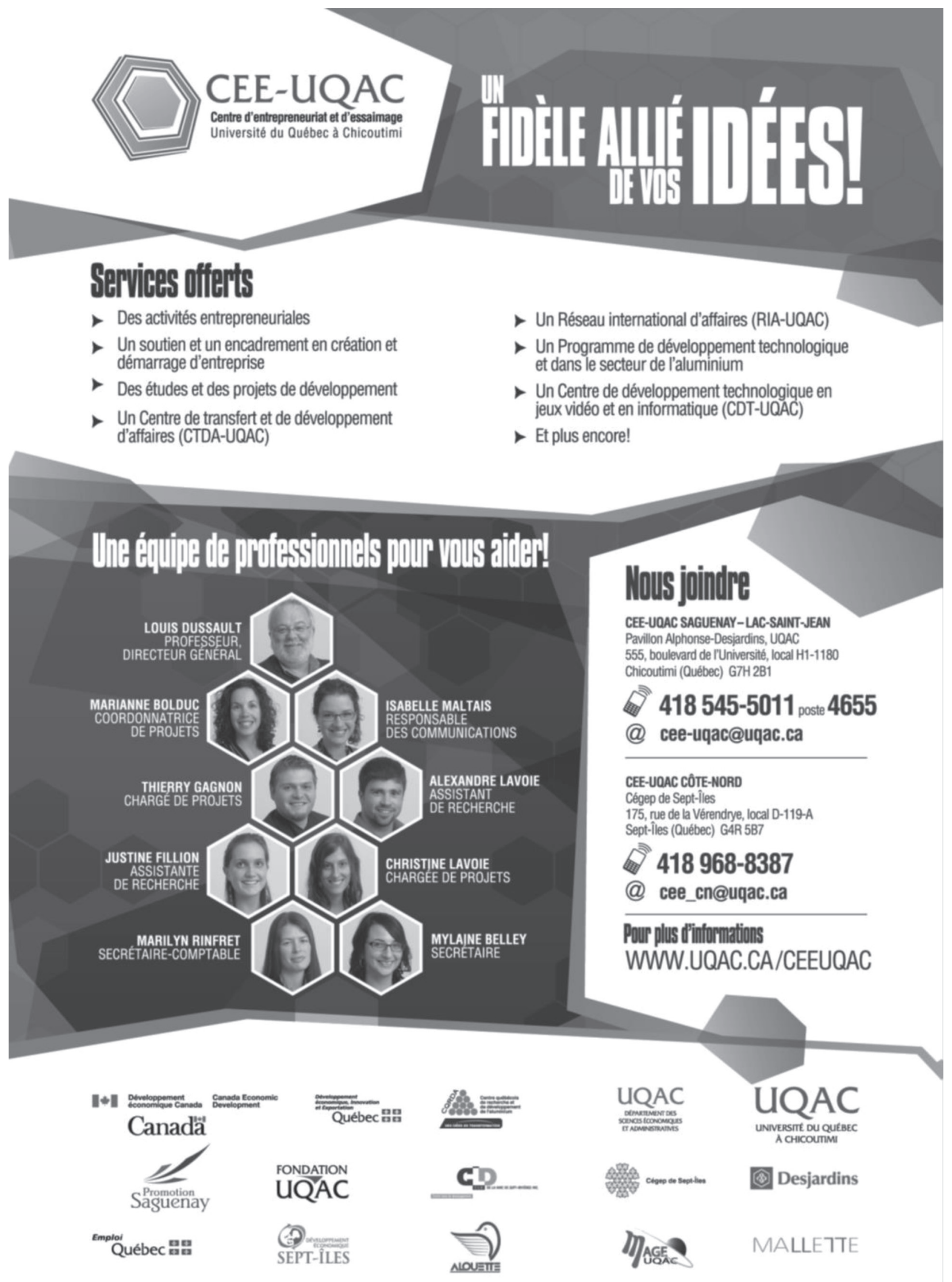

